\title{
Chapter 1 \\ Control of a Magnus Effect-Based Airborne Wind Energy System
}

\author{
Ahmad Hably, Jonathan Dumon, Garrett Smith and Pascal Bellemain
}

\begin{abstract}
This chapter studies the control of an airborne wind energy (AWE) system that uses a rotating cylinder to provide aerodynamic lift with the Magnus effect. During a production phase, the Magnus cylinder turns through a tether an on-ground electrical generator. During a recovery phase, the on-ground generator becomes a motor in order to return the Magnus cylinder to its starting position. The proposed control strategy aims at stabilizing the output power production which can be used for example in AWE off-grid applications. Three case studies are presented. In the first case study, a small scale system in a wind tunnel setup is presented. The proposed control scheme is applied numerically and experimentally. The controller works well to effectively manage the tether length. However, a comparison of the results demonstrates the penalizing effects of wind turbulence with a factor of three difference in power production. In the second case study, the control strategy is numerically applied to a medium scale prototype with a potential power rating of 50 $\mathrm{kW}$. The case study shows that the control strategy is very effective to track the desired power production even in the presence of fluctuations in the magnitude of the wind velocity. In the third case study, the scalability of the system is evaluated by numerically applying the control scheme on a MW scale platform. Results show that the system with a span equal to the diameter of a conventional wind turbine can generate an equivalent amount of power.
\end{abstract}

Ahmad Hably $(\varangle) \cdot$ Jonathan Dumon · Pascal Bellemain

Grenoble Institute of Technology, GIPSA-Lab, 38000 Grenoble, France

e-mail: ahmad.hably@gipsa-lab.grenoble-inp.fr

Garrett Smith

Wind Fisher S.A.S., 2 allee du Vivarais, 31770 Colomiers, France 


\subsection{Introduction}

The concept of airborne wind energy (AWE) has attracted a lot of interest in the last few years [Cherubini2015]. In an interview in June 2015, Bill Gates said that he is planning to invest more than two billion dollars in green technologies and highlighted promising areas of research that "involve among others kites, kite-balloon hybrids known as kytoons or flying turbines" [Independent2015].

AWE systems replace the blades of conventional wind turbines by a controlled flying wing that captures the energy of the wind. EnerKite claims that their AWE system doubles the output whilst saving 95\% of resources [enerkite2014]. This concept of producing more energy with less material goes in the direction of recent movement of frugal innovation that promotes "How to do more with less".

AWE systems can be divided into two main classes depending on the location where energy is produced:

- Systems using the lift mode as introduced in [Loyd1980] where the mechanical power is transferred to the ground. Energy is produced during a production phase, in which the aerodynamic lifting device is pulled by the wind while unrolling the tether from a drum which turns an on-ground electrical generator. This phase is followed by a recovery phase that begins when the tether reaches its predefined maximum length, and hence needs to be reeled-in, an operation that consumes energy. These on-ground systems are studied, for example by Kitegen [Canale2009], and Ampyx Power [AmpyxPowerBV].

- On-board production using the drag mode as introduced in [Loyd1980]. The generator is embedded in the airborne structure and electric energy is produced in-flight and transferred to the ground using conducting tethers. This type of systems is investigated, for example by Makani Power [Makani2016].

Most of the aforementioned systems use either flexible or rigid wings as aerodynamic lifting devices. To increase the traction force during reel-out or the energy harvesting in drag mode, the wing is operated in crosswind maneuvers. On the other hand, Omnidea Lda have used a Magnus effect-based system in its High Altitude Wind Energy project (HAWE) [Omnidea2013]. The operation principle of their platform is based on the traction force of a rotating cylindrical balloon employing both aerostatic as well as aerodynamic lift mechanisms [Penedo2013, Pardal2015]. Magnus effect-based airborne wind energy systems generate an aerodynamic lift that depends on the apparent wind speed at the Magnus cylinder and its angular speed. Electrical energy is produced as for on-ground systems, with the difference that the balloon is not operated in crosswind flight maneuvers. The fundamental reason is that a cylindrical body will always have a significant aerodynamic drag because of its flow cross section. As a consequence, the lift-to-drag ratio is limited to comparatively low values which leads to relatively low performance when operated in crosswind mode despite of its high lift coefficient. On the other hand, Magnus effect-based system offers a huge advantage of being lighter than air, which greatly simplifies the takeoff and landing phases when the wind is insufficient. In addition, as opposed to the AWE systems using airfoil kites or wings whose lift 
and drag vector magnitudes depend on the angle of attack measured between airfoil chord and apparent wind velocity, Magnus effect-based systems only change lift and drag vector direction, and not magnitude, when there is a change in apparent wind direction.

In [Perkovic2013], the feasibility of the Magnus effect-based concept has been studied. In [Milutinovic2015], the control variables were optimized for an airborne wind energy production system showing optimal vertical trajectories.

In the present chapter, using similar vertical trajectories, the proposed control strategy aims at controlling the power produced by a Magnus effect-based AWE system. The on-ground generator controls the tether length and the cycle period. The performance of the control strategy is satisfying even in the presence of highly perturbed wind speeds.

The chapter is organized as follows. In Sect. 1.2 the system model is introduced. The control strategy presented in Sect. 1.3 applies to the experimental platform described in Sect. 1.4 where both simulation and experimental results are shown. A numerical application of the proposed control strategy on a medium scale system based on Omnidea's experimental platform is presented in Sect. 1.5. Some numerical results on a MW scale system are presented in Sect. 1.6. The chapter ends with some conclusions and perspectives in Sect. 1.7.

\subsection{System Modeling}

The airborne wind energy system studied is composed of a light-weight rotating cylindrical lifting device, called hereafter the Magnus rotor, that supplies a traction force to an on-ground generator through a tether. This device generates aerodynamic lift and drag forces. Lift contribution comes from aerodynamic lift, the well-known Magnus effect, and aerostatic lift, by using Helium as a filling gas. The resultant traction force is transferred via the tether to the on-ground generator where a drum is used to convert the linear motion of the tether into shaft power, which is used to drive a generator. For recovery, this operation is reversed, i.e. the generator is operated as a motor and the aerodynamic force of the Magnus rotor is reduced by switching off the rotation. Acting on the Magnus rotor, as shown in Fig. 1.1, are the aerodynamic force $\mathbf{F}_{a}$, which can be split up into a aerodynamic lift $\mathbf{L}$ and a drag $\mathbf{D}$ component, the gravitational force on the Magnus rotor $\mathbf{P}$, the buoyancy force $\mathbf{B}$ and the tensile force $\mathbf{T}$ in the tether.

Aerodynamic lift and drag forces can be expressed by:

$$
L=\frac{1}{2} \rho S v_{a}^{2} C_{L}, \quad D=\frac{1}{2} \rho S v_{a}^{2} C_{D}
$$

where $\rho$ is the air density, $v_{a}$ is the apparent wind velocity, $S$ is the Magnus rotor projected surface area in the direction of the apparent wind velocity, $C_{L}$ and $C_{D}$ are aerodynamic lift and drag coefficients respectively. For Magnus effect-based systems, aerodynamic lift coefficient $C_{L}$ and drag coefficient $C_{D}$ are functions of the 


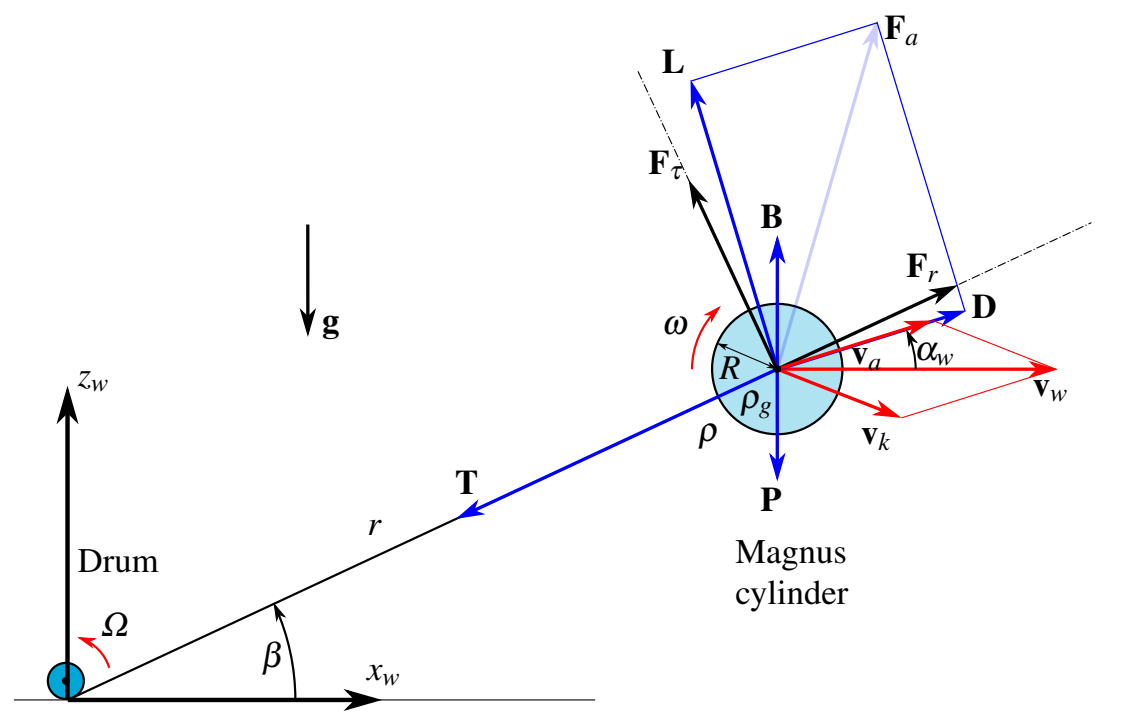

Fig. 1.1 The different forces acting on the Magnus effect-based airborne wind energy system, the translation velocity of the Magnus rotor $v_{k}$ and its angular velocity $\omega$, the angular velocity of the drum $\Omega=\dot{r} / R_{d}$ and the wind velocity with respect to the ground $v_{w}$.

spin ratio $X$ [Seifert2012]. The Magnus rotor spin ratio is given by the following equation:

$$
X=\frac{\omega R}{v_{a}}
$$

where $\omega$ is the Magnus rotor angular velocity and $R$ is the Magnus rotor radius. The buoyancy force can be calculated from Archimedes' principle:

$$
B=\rho V_{0} g
$$

where $V_{0}$ is the volume of the Magnus rotor and $g$ is the gravitational acceleration. The combined mass of all airborne system components (Magnus rotor, contained gas, rotor drive train plus tether and bridle lines) is denoted by $M_{M}$ :

$$
M_{M}=M+V_{o} \rho_{g}+M_{l} r
$$

where $M$ is the mass of the airborne structure, $M_{l}$ denotes the mass per tether length and $\rho_{g}$ is the gas density. The wind velocity $\mathbf{v}_{w}$ is assumed to be parallel to ground.

The apparent wind velocity $v_{a}$ is defined by:

$$
\mathbf{v}_{a}=\mathbf{v}_{w}-\mathbf{v}_{k}
$$

where $\mathbf{v}_{k}$ is the translation velocity of the Magnus rotor. In this study, the movement of the Magnus rotor is assumed to be in the vertical plane. It is also assumed that the tether of length $r$ is always in tension and forms a straight line. It has an 
elevation angle $\beta$ with respect to the ground plane. Furthermore, the cylindrical lifting device does not allow the definition of an angle of attack in the cross sectional plane. In order to find the dynamic model, fundamental dynamic equations are used. Considering the two translational degrees of freedom of the system, $r$ and $\beta, \mathbf{v}_{k}$ can be decomposed into a radial velocity component $v_{k, r}=\dot{r}$ and a tangential velocity component $v_{k, \tau}=r \dot{\beta}$. Differentiation of $\mathbf{v}_{k}$ with respect to time yields the radial acceleration component $d v_{k, r} / d t=\ddot{r}-r \dot{\beta}^{2}$ and the tangential acceleration component $d v_{k, \tau} / d t=r \ddot{\beta}+2 \dot{r} \dot{\beta}$. The resultant forces $F_{r}$ and $F_{\tau}$ on the Magnus rotor are respectively the radial and tangential force components according to the polar coordinate system $(r, \beta)$ as shown in Fig. 1.1.

$$
\begin{aligned}
& F_{r}=-T+L \sin \left(\beta-\alpha_{w}\right)+D \cos \left(\beta-\alpha_{w}\right)-P \sin \beta+B \sin \beta \\
& F_{\tau}=L \cos \left(\beta-\alpha_{w}\right)-D \sin \left(\beta-\alpha_{w}\right)-P \cos \beta+B \cos \beta
\end{aligned}
$$

where $\alpha_{w}$ is the angle that the apparent wind velocity forms with the horizontal. The dynamic model can then be derived in 2D polar coordinates:

$$
\begin{aligned}
\ddot{\beta} & =\frac{1}{r}\left[-2 \dot{\beta} \dot{r}+\frac{F_{\tau}}{M_{M}}\right] \\
\ddot{r} & =\frac{1}{M_{M}+M_{D}}\left[r \dot{\beta}^{2} M_{M}+F_{r}\right]
\end{aligned}
$$

where $M_{D}=I / R_{d}^{2}$ with moment of inertia of the on-ground generator $I$ and its radius $R_{d}$. In addition to these equations, we add the dynamics of the on-ground generator:

$$
\dot{T}=\beta_{T}\left(u_{T}-T\right)
$$

where $u_{T}$ is the desired traction force and $\beta_{T}$, homogeneous to a frequency, represents its dynamic response modeled here as a first order dynamic system.

\subsection{Control Strategy}

The control strategy to be applied to the Magnus-based system aims at stabilizing the mean output power produced during a given cycle (recovery phase then production phase). The tether traction force $T$ and its speed $\dot{r}$ are forced to track some reference signals related to a desired reference power $P_{r e f}$ to be produced. For simplicity, $P_{r e f}$ is assumed to be constant, however the control strategy can be adapted to varying $P_{\text {ref }}$ as shown later.

During the cycle, the Magnus rotor moves from a minimum radial position $r_{\text {min }}$ to a maximum radial position $r_{\max }$ at a reel-out speed $\dot{r}_{\text {prod }}$ during production phase and from $r_{\max }$ to $r_{\min }$ at a negative reel-in speed $\dot{r}_{\text {rec }}$ during the recovery phase. Since $\dot{r}_{\text {prod }}$ and $\dot{r}_{\text {rec }}$ are assumed to be constant, the proposed algorithm tracks $P_{r e f}$ 
by controlling the traction force $T$. A given cycle is defined by the time period from the beginning of the recovery phase to the end of the production phase. The recovery phase starts at time $t_{0}$ and ends at time $t_{1}$. Then the production phase starts at time $t_{1}$ and ends at time $t_{2}$ (see Fig. 1.2). Time $t_{1}$ can be calculated by

$$
t_{1}=t_{0}+\frac{\left(r_{\text {max }}-r_{\text {min }}\right)}{-\dot{r}_{\text {rec }}}
$$

Time $t_{2}$ can be calculated by

$$
t_{2}=t_{1}+\frac{\left(r_{\text {max }}-r_{\text {min }}\right)}{\dot{r}_{\text {prod }}}
$$

In order to produce a net output power equals to $P_{r e f}$, the output energy to be produced during a cycle $E_{r e f}$ is given by $P_{r e f}\left(t_{2}-t_{0}\right)$. During the cycle, the output

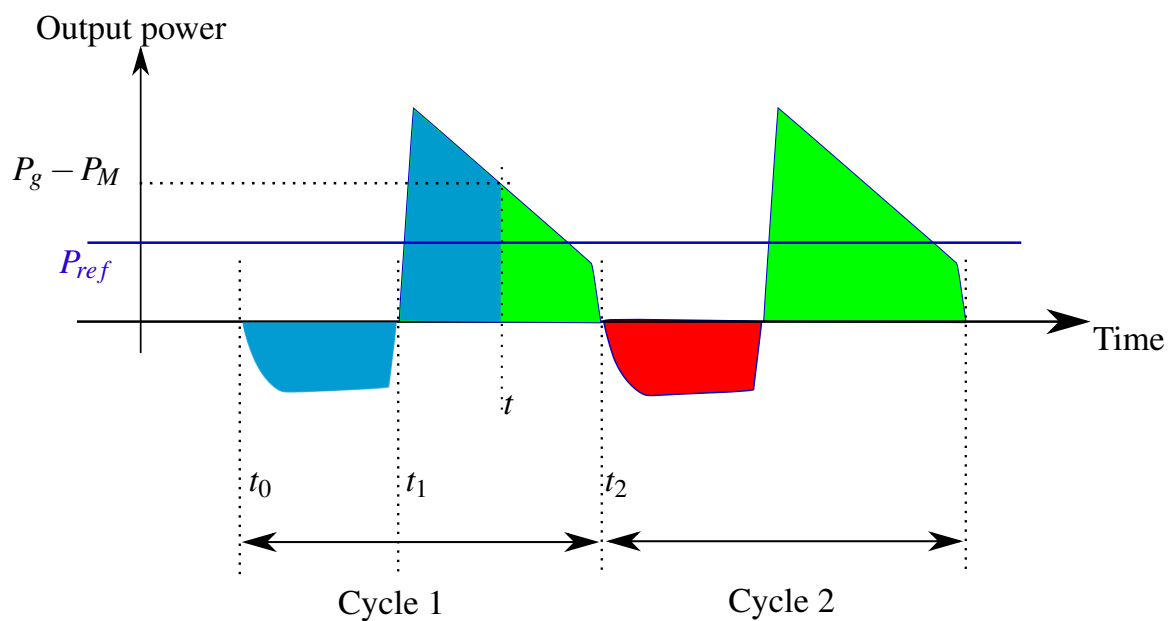

Fig. 1.2 A sketch of the instantaneous output power as a function of time covering several pumping cycles. For a cycle, the red area $E_{\text {rec }}$ represents the energy consumed during the recovery phase between time $t_{0}$ and time $t_{1}$ and the green area $E_{\text {prod }}$ represents the energy produced during the production phase between time $t_{1}$ and time $t_{2}$. The blue area represents the output energy at time $t$ for the beginning of the cycle. Desired reference power $P_{r e f}$ is assumed to be constant.

energy produced from time $t_{0}$ to time $t$ is calculated by

$$
E=\int_{t_{0}}^{t}\left(P_{g}-P_{M}\right) d t
$$

where $P_{g}$ is the produced output power of the on-ground generator and $P_{M}$ is the power consumed by the Magnus motor. In order to satisfy $E=E_{r e f}$ at the end of the cycle, the remaining energy to be produced $E_{\text {prod }}$ from time $t$ to time $t_{2}$ has to satisfy: 


$$
E_{\text {prod }}=E_{\text {ref }}-E
$$

Subsequently, the reference traction force has to satisfy for $t \in\left[t_{0}, t_{2}\right]$

$$
T_{\text {ref }}=\frac{1}{\dot{r}} \frac{E_{\text {prod }}}{\left(t_{2}-t\right)}
$$

As $T$ cannot be negative, $T_{r e f}$ is set to zero for $t \in\left[t_{0}, t_{1}\right]$.

In order to implement the proposed control strategy, two other controllers $K_{1}$ and $K_{2}$ are used as shown in Fig. 1.3. The tether length is controlled by $K_{1}$ through the desired traction force $u_{T}$ of the on-ground generator. In order to track $T_{r e f}$ obtained from controller $K_{3}, K_{2}$ controls spin ratio $X$ of the Magnus rotor. Controller $K_{3}$ is given in Eq. (1.15). Controllers $K_{1}$ and $K_{2}$ are classical PID controllers in parallel form whose parameters are tuned empirically with the following constraints:

- $K_{2}$ is set to have a fast response time to get $T_{r e f}=T$.

- $K_{1}$ is set to have a faster response time than $K_{2}$ in order to have a decoupled control between the tether length $r$ and the traction force $T$.

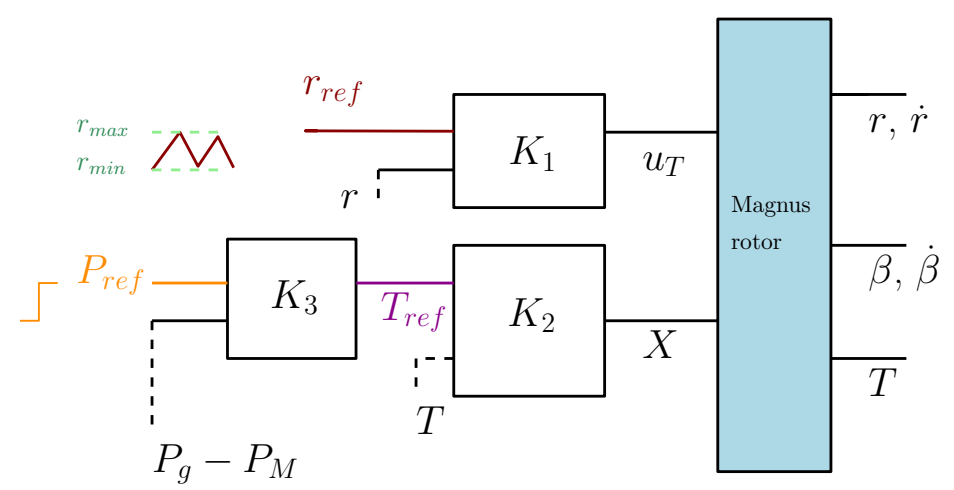

Fig. 1.3 An overview of the proposed control system. Three controllers $K_{1}, K_{2}$, and $K_{3}$ are used. The Magnus rotor moves from minimum radial position $r_{\min }$ to a maximum radial position $r_{\max }$. $T_{r e f}$ is obtained by Eq.(1.15). $X$ is the spin ratio and $u_{T}$ is the desired traction force.

In the rest of this chapter, three case studies will be presented. In the first case, the control will be applied numerically and experimentally on a small scale indoor system. In the second case, we will numerically study a medium scale system. In the last case, the control strategy will be numerically applied to a MW scale system. 


\subsection{Control of a Small Scale Laboratory Test Setup}

In this section, the control of a small scale wind tunnel setup will be presented. It is based on Gipsa-lab's experimental setup. This experimental setup has been used in [Hably2013] and [Lozano2013]. This setup gives us some flexibility and allows us to test our prototypes and the proposed control strategies independently of the weather conditions. It is composed of a wind tunnel, the Magnus rotor, and the ground station.

\subsubsection{Wind Tunnel}

The fan section of the wind tunnel is composed of nine brushless electrical motors equipped with two-blade fans of $0.355 \mathrm{~m}$ diameter, see Fig. 1.4 (top left). These motors, $800 \mathrm{~W}$ each, are distributed on a tunnel cross section area of $1.85 \mathrm{~m}^{2}$. The air flow first passes through a honeycomb then in a tunnel of $1.8 \mathrm{~m}$ length in order to stabilize it. A hot wire wind speed sensor ${ }^{1}$ is used to measure the airspeed. The output air flow speed can reach $9 \mathrm{~m} / \mathrm{s}$ with a typical standard deviation of 0.18 measured at $1 \mathrm{~Hz}$. As there are fast variations, the air flow can be better characterized with a smaller sample time.

We choose to use nine hobbyist propulsion sets of electrical motors because this option turns out to be cheaper than the use of a single $7.2 \mathrm{~kW}$ motor with a driver and a propeller. This design of an open wind tunnel was chosen based on economic reasons at the expense of flow quality that can be provided by a closed wind tunnel architecture.

\subsubsection{Magnus Rotor}

The Magnus rotor used in the experimental setup is a light-weight cylinder built with carbon rods, polystyrene and transparent plastic paper, see Fig. 1.3 (bottom left). The rotation of the Magnus rotor is provided by a mini DC motor mounted on one end of it. Its current control and speed sensor are implemented with a homemade driver. The parameters of the Magnus rotor are given in Table 1.1. The size of this Magnus rotor does not allow us to have a lighter-than-air system so it is not filled with Helium. The rigid frame design proposed allows us to have a better balanced symmetrical structure than if we have used a textile structure.

\footnotetext{
${ }^{1}$ Measurement frequency of $1 \mathrm{~Hz}$ achievable with serial interface.
} 


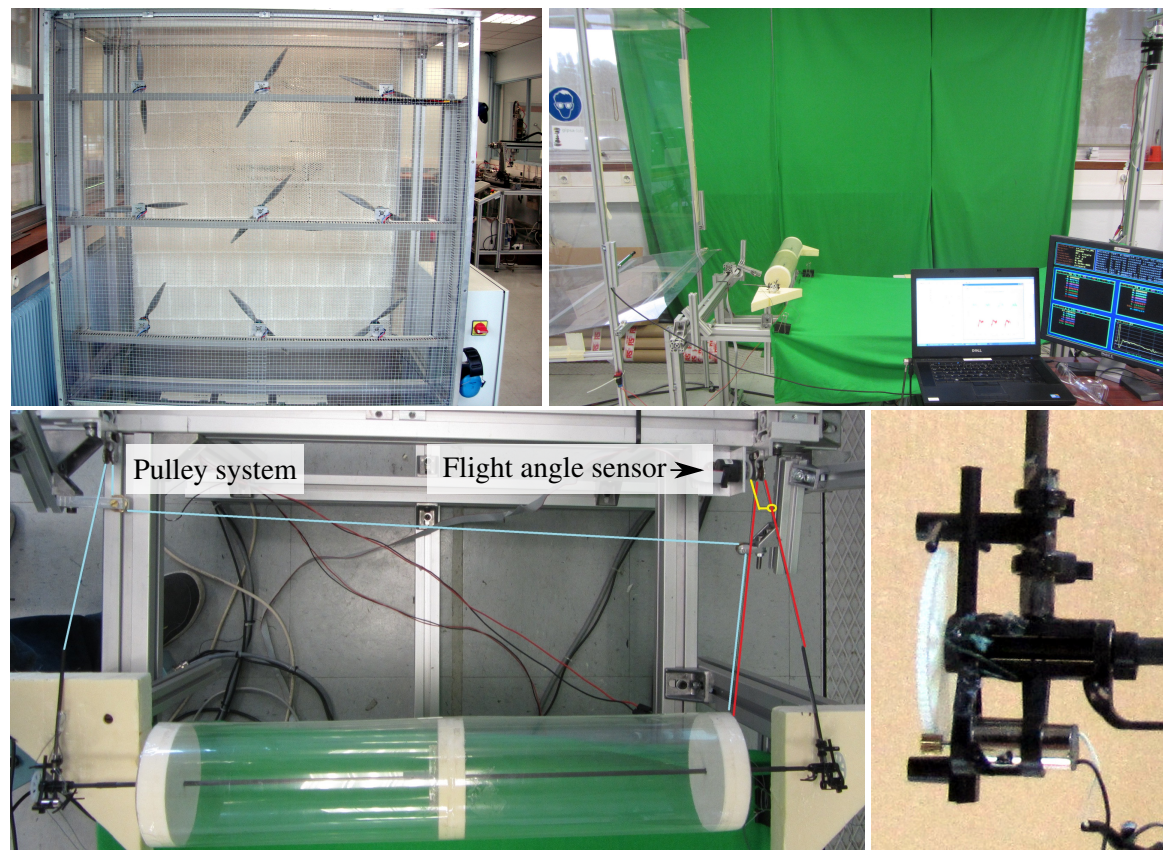

Fig. 1.4 Wind tunnel setup: front view of fan section (top left), side view of test section with computer hardware in foreground (top right), Magnus rotor (bottom left) and DC motor used to rotate the Magnus rotor (bottom right).

Table 1.1 Parameters of the test setup.

\begin{tabular}{lll}
\hline Symbol & Name & Value \\
\hline$M_{M}$ & Mass of airborne subsystem & $0.11 \mathrm{~kg}$ \\
$M_{l}$ & Mass per tether length & $0 \mathrm{~kg} / \mathrm{m}$ (neglected) \\
$R$ & Magnus rotor radius & $0.047 \mathrm{~m}$ \\
$L_{m}$ & Magnus rotor length & $0.45 \mathrm{~m}$ \\
$M_{D}$ & Ground station rotor mass & $0.0481 \mathrm{~kg}$ \\
$R_{d}$ & Drum radius & $0.05 \mathrm{~m}$ \\
$\rho$ & Air density & $1.225 \mathrm{~kg} / \mathrm{m}^{3}$ \\
$\beta_{T}$ & Inverse of time constant of motor current $14.28 \mathrm{~s}^{-1}$ \\
& loop & $4 \times 10^{4}$ \\
\hline
\end{tabular}

\subsubsection{Ground Station}

The ground station is composed of a dynamo-motor system Maxon 2260L DC $100 \mathrm{~W}$ driven by a four-quadrants amplifier Maxon ADS 50/10 that controls current 
through the motor. Two incremental encoders provide measurement of the elevation angle $\beta$ and tether length $r$. Control references of DC motors are sent to drivers with a DAC PCI DAS1200 from Measurement Computing and a torque sensor provides an accurate measurement of tether tension. Controllers are implemented on the experimental setup using the xPC target real-time toolbox of Matlab, see Fig. 1.3 (top right).

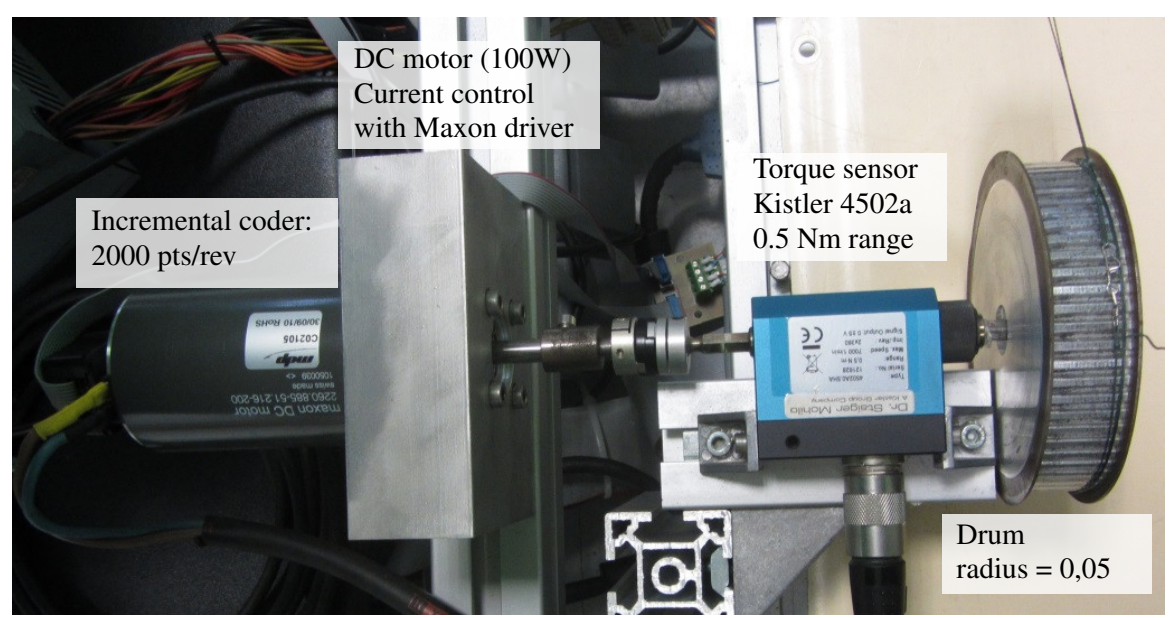

Fig. 1.5 The ground station. The drum is made for a tooth belt, but used here with a line.

\subsubsection{Identification}

In a first place, the response time of the DC motor used to rotate the Magnus rotor is identified (see Fig1.6). The second step is to identify, by regression of the measured data, the aerodynamic lift and drag coefficients as functions of the spin ratio. The obtained results are comparable to the theoretical results [White2015] for Reynolds number $\mathrm{Re}=3.8 \times 10^{4}$ and used in [Milutinovic2015] (see Fig. 1.7). The identified model for the aerodynamic lift and drag coefficients function of spin ratio $X$ is:

$$
\begin{aligned}
& C_{D}=0.73 X^{2}-1.2 X+1.2131 \\
& C_{L}=0.0126 X^{4}-0.2004 X^{3}+0.7482 X^{2}+1.3447 X-0.2
\end{aligned}
$$

The last step in the identification phase is to find the operational limits of our platform. We have noticed that friction in the pulleys is significant. The increase of mechanical friction forces is a well known physical phenomenon when scaling down. We have measured the tension in the tether as a function of the tether length $r$ for different angular speeds $\omega$ of the Magnus rotor and the tether speed $\dot{r}$. We have 
Fig. 1.6 The dynamic re-

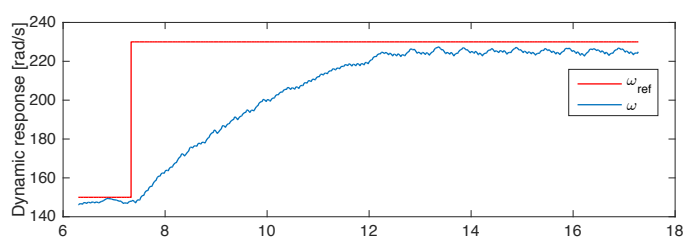
sponse of the DC motor to a step signal for a wind speed $v_{w}=0 \mathrm{~m} / \mathrm{s}$. In blue is the measured angular speed and in red the reference angular speed (top). The power consumed for this step signal is also shown (bottom).

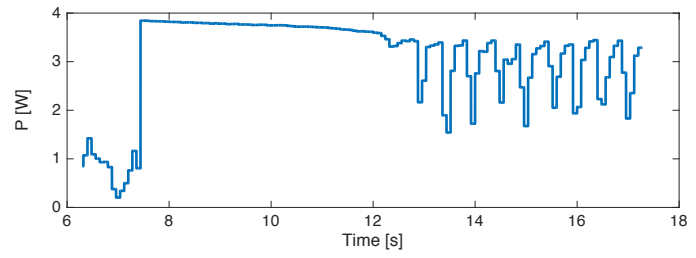

Fig. 1.7 Aerodynamic Lift and drag coefficients identification as functions of the spin ratio $X$. Wind speed $v_{w}$ varies

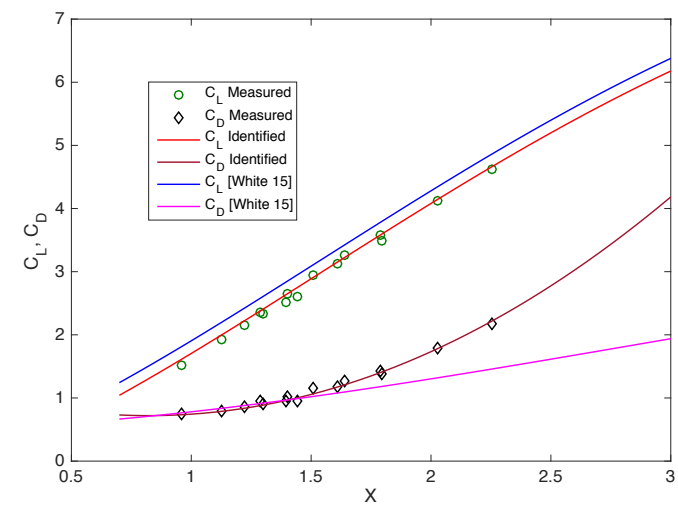
from $4.76 \mathrm{~m} / \mathrm{s}$ to $7.26 \mathrm{~m} / \mathrm{s}$.

found our platform can provide a limited difference of traction force that one can use to produce energy. This is shown in the difference between the upper and lower zones of Fig. 1.8.

\subsubsection{Simulation Results}

In this section, the proposed control strategy is tested numerically in a simulation environment using Eqs. (1.8), (1.9), and (1.10). Our objective is to test the control strategy and to have a cycle with a positive net energy output. The following conditions are used.

- Reynolds number is $4 \times 10^{4}$. Wind speed $v_{w}=6.28 \mathrm{~m} / \mathrm{s}$.

- The minimum tether length is $r_{\min }=0.1 \mathrm{~m}$ and its maximum is $r_{\max }=0.7 \mathrm{~m}$. These limits are imposed by the wind window of the wind tunnel.

- The tether speed in the production phase is $\dot{r}_{\text {prod }}=0.1 \mathrm{~m} / \mathrm{s}$ and in the recovery phase $\dot{r}_{r e c}=-0.1 \mathrm{~m} / \mathrm{s}$. These values, even far from the optimal value $\left(v_{w} / 2\right)$ 
Fig. 1.8 The measured tension in the tether as a function of the tether length $r$ for different angular speeds $\omega$ of the Magnus rotor and tether speed $\dot{r}$, for a wind speed $v_{w}=6.2 \mathrm{~m} / \mathrm{s}$. The zone A is the possible force difference that can be used to produce energy. This zone is reduced to zone B due to the pulleys friction. This gives an idea of the feasibility of a positive power production cycle and what one could potentially get if this friction is reduced.

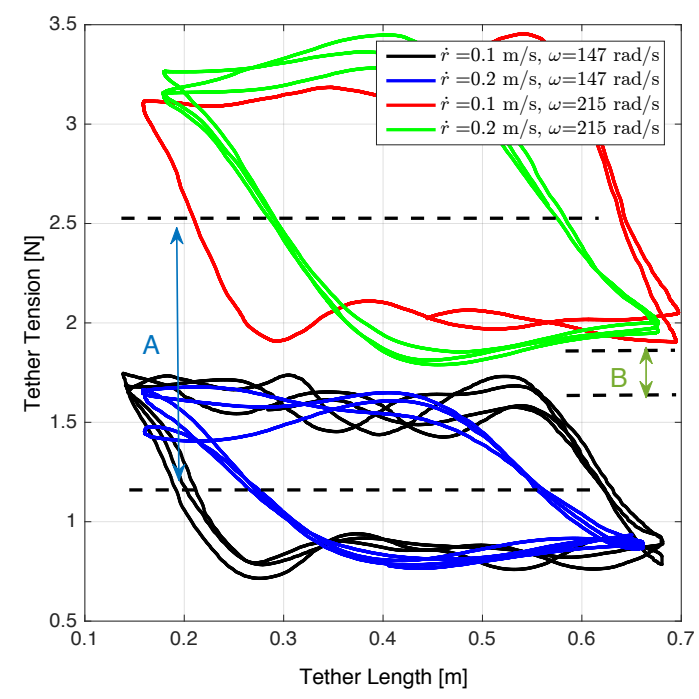

according to the main theory of simple kite, Fig. 2 of [Loyd1980], were imposed again by the limits of the wind window.

- Friction identified in Fig. 1.8 leads to a limited range of power production. For this reason, we choose to deactivate the controllers $K_{2}$ and $K_{3}$ using constant values of spin ratio $X$.

- We have chosen to control only $\omega$ which gives an average value of $X$. A constant angular speed of the Magnus rotor is used in the production phase $\omega_{\text {prod }}=200$ $\mathrm{rad} / \mathrm{s}$ and in the recovery phase $\omega_{\text {rec }}=140 \mathrm{rad} / \mathrm{s}^{2}$. Considering that $\dot{r}$ is negligible with respect to $v_{w}$ and simple kite conditions ( $\mathbf{v}_{\mathbf{k}}$ and $\mathbf{T}$ are colinear), this leads to $v_{a} \approx v_{w}=6.28 \mathrm{~m} / \mathrm{s}$. The spin ratio is then $X_{\text {prod }}=1.5161$ in the production phase and $X_{\text {rec }}=1.0613$ in the recovery phase.

The tether length follows the desired radial position as shown in Fig. 1.9. As expected, the traction force increases as the angular Magnus rotor speed increases. The movement in the vertical plane is shown in Fig. 1.10. The application of this control strategy enables us to produce a positive net energy output as shown in Fig. 1.11.

\footnotetext{
${ }^{2}$ A zero rotating velocity during the recovery phase cannot be set because of the limits of Gipsalab's experimental setup.
} 

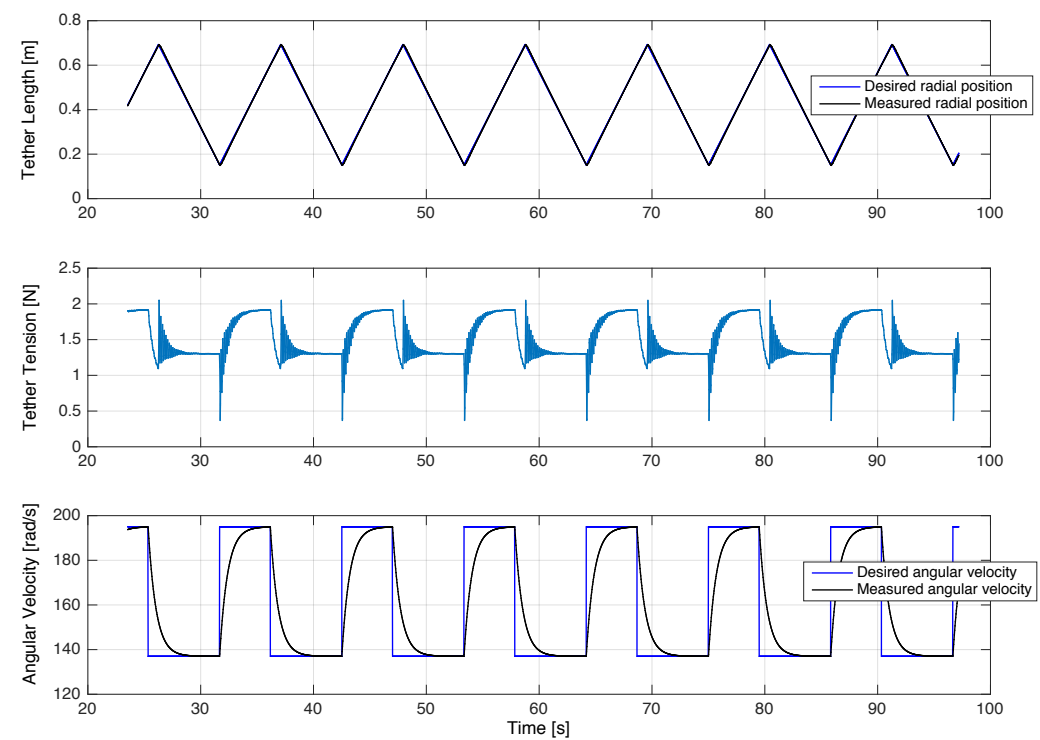

Fig. 1.9 Tether length, tether tension and the angular speed of the Magnus rotor as function of time in the simulation of the small scale system for a wind speed $v_{w}=6.28 \mathrm{~m} / \mathrm{s}$. The oscillation in the tether tension is due to the choice of controller parameters.

Fig. 1.10 The production cycles in the simulation of the small scale system for a wind speed $v_{w}=6.28 \mathrm{~m} / \mathrm{s}$. The direction of the arrows indicates the movement of the Magnus rotor: Green for the production phase and red for the recovery phase.

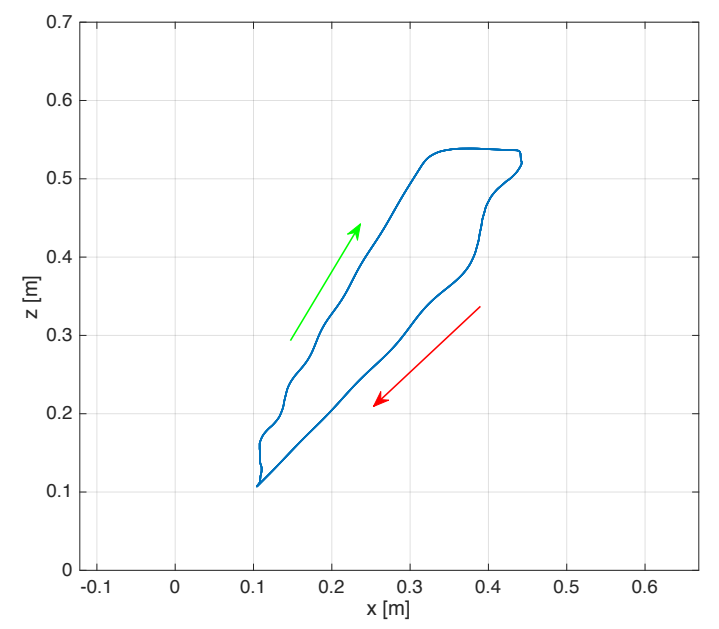

\subsubsection{Experimental Results}

Using the same conditions as in the previous simulation section, the control strategy is applied to the experimental setup (Figs. 1.12-1.14). A movie that shows the experiment can be found on our website [GipsaWeb]. 


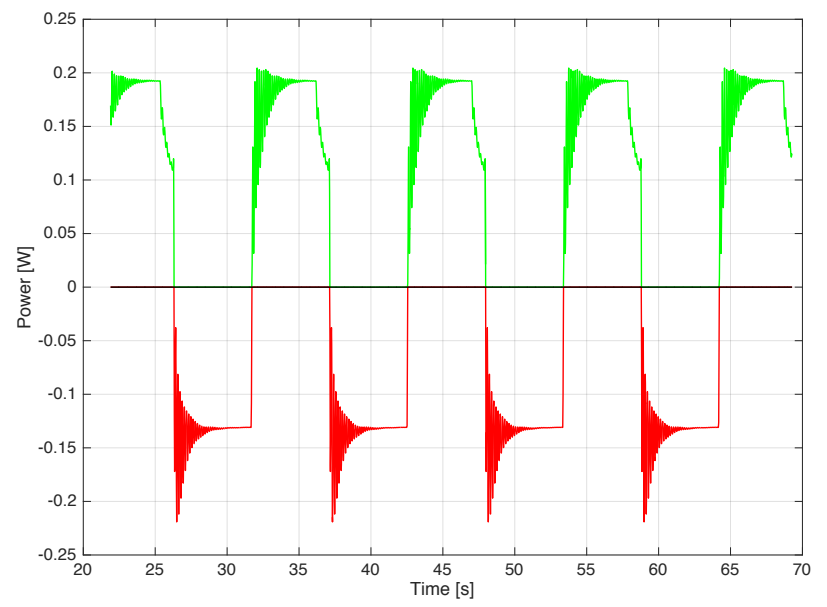

Fig. 1.11 The power produced in the simulation of a small scale system. The net output power is $0.0327 \mathrm{~W}$. Wind speed $v_{w}=6.28 \mathrm{~m} / \mathrm{s}$
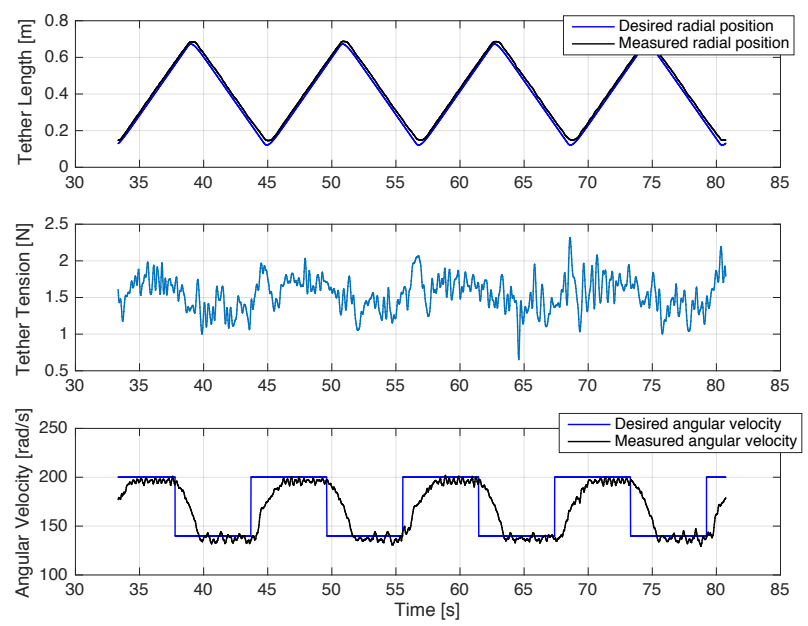

Fig. 1.12 Tether length, tether tension and the angular speed of the Magnus rotor as function of time in the experimentation on the small scale system. The measured wind speed $v_{w}=6.28 \mathrm{~m} / \mathrm{s}$ with a standard deviation of 0.184 .

\subsubsection{Results Discussion}

From the simulation results, one can see that after stabilizing the angular velocity $\omega$, the elevation angle $\beta$ also stabilizes confirming simple kite conditions. In the experimental results, there are significant oscillations of $\beta$ due to wind turbulence of the used wind tunnel. As a consequence, simple kite conditions are not verified. 
Fig. 1.13 The measured production cycles of the small scale system versus the simulation production cycles using the same wind data. The measured wind speed $v_{w}=6.28 \mathrm{~m} / \mathrm{s}$ with a standard deviation of 0.184 . The direction of the arrows indicates the movement of the Magnus rotor: Green for the production phase and red for the recovery phase.
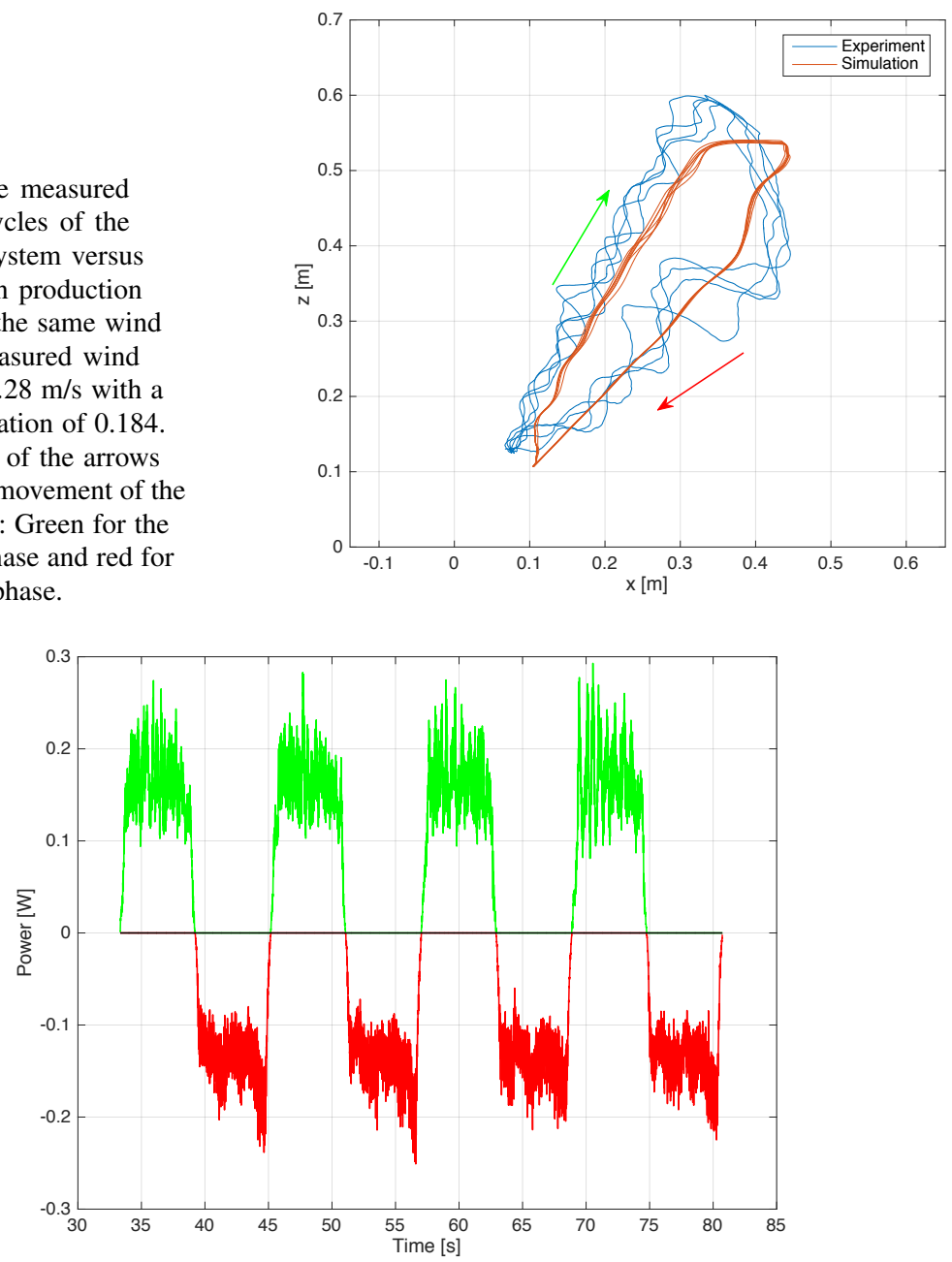

Fig. 1.14 The power produced in the experimentation on the small scale system. The net output power is $0.0099055 \mathrm{~W}$. The measured wind speed $v_{w}=6.28 \mathrm{~m} / \mathrm{s}$ with a standard deviation of 0.184 .

Moreover, these oscillations impact the tether tension as shown in Fig. 1.12 and the produced power shown in Fig. 1.14, which is reduced by a factor of three compared to the simulation results. Nevertheless, the similar shapes of the cycles shown in Fig.1.13 gives us an idea on the validity of the proposed model. For this small scale system, the power consumed by the Magnus motor $P_{M}$ is much larger than the power produced by the system due, among others, to the significant effect of frictions.

For larger scale systems, frictions become less important compared to aerodynamic forces. Wind turbulence will also produce less impact on elevation angle $\beta$ due to length of the tether and to larger inertia of the airborne subsystem. One has 
to adjust the dynamics of the on-ground generator and the Magnus motor in order to match the time scale of production cycles. In addition, a model of aerodynamic lift and drag coefficients must be adapted to the considered Reynolds number. Finally, as the volume increases with the cube of the cylinder dimensions and the mass increases with the square since it is related to the surface, scaling up the system allows to get a lighter-than-air structure by filling it with lighter-than-air gas like Helium.

\subsection{Numerical Application to a Medium Scale System}

The complete control strategy has been numerically applied to Omnidea's system shown in Fig. 1.15. We have used the dimensions of the Magnus rotor presented in [awec2015]. This Magnus rotor is filled with Helium.

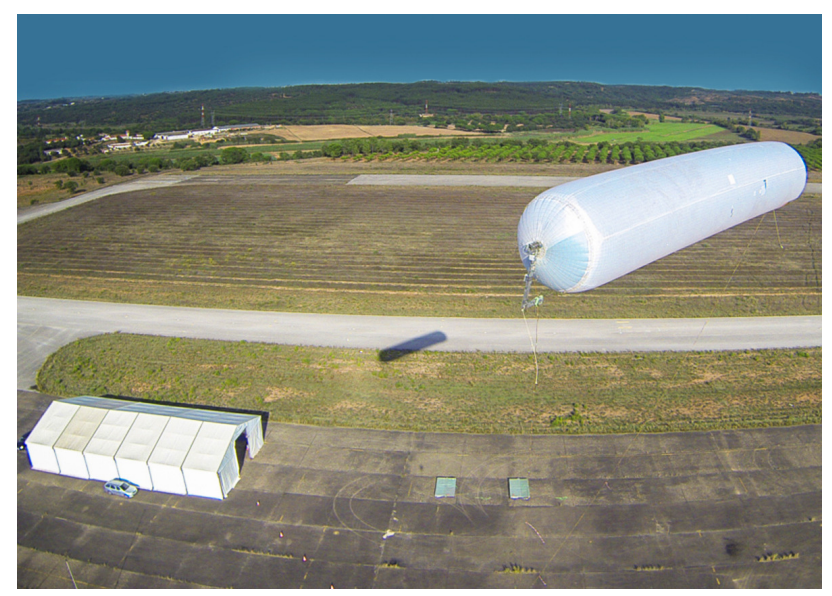

Fig. 1.15 Omnidea's platform during experiments [Omnidea2013].

Its parameters are listed in Table 1.2. The aerodynamic lift and drag coefficients used are those presented in [White2015] for Reynolds number $\operatorname{Re}=3.8 \times 10^{4}$ :

$$
\begin{aligned}
& C_{D}=-0.0211 X^{3}+0.1837 X^{2}+0.1183 X+0.5 \\
& C_{L}=0.0126 X^{4}-0.2004 X^{3}+0.7482 X^{2}+1.3447 X
\end{aligned}
$$

Note that for a wind speed $v_{w}=10 \mathrm{~m} / \mathrm{s}$, Reynolds number is $\operatorname{Re}=1.7 \times 10^{6}$. For higher values of $\operatorname{Re}$, the $C_{D}$ expression of the above equation can be slightly different.

In order to implement the proposed control strategy, we choose to reproduce a vertical trajectory similar to those suggested in [Milutinovic2015] that we will reduce its efficiency in order to stabilize a desired power produced $P_{\text {ref }}$. We have determined the feasibility regions for $r_{\min }=200 \mathrm{~m}$ and $r_{\max }=300 \mathrm{~m}$. For a wind 
Table 1.2 Parameters of the medium scale Magnus rotor.

\begin{tabular}{lll}
\hline Symbol & Name & Value \\
\hline$M_{M}$ & Mass of airborne subsystem & $91.22 \mathrm{~kg}$ \\
$R$ & Magnus rotor radius & $1.25 \mathrm{~m}$ \\
$L_{m}$ & Magnus rotor length & $16 \mathrm{~m}$ \\
$\rho_{H e}$ & Helium density & $0.1427 \mathrm{~kg} / \mathrm{m}^{3}$ \\
$\rho_{\text {air }}$ & Air density & $1.225 \mathrm{~kg} / \mathrm{m}^{3}$ \\
$M_{l}$ & Mass per tether length & $0.2 \mathrm{~kg} / \mathrm{m}$ \\
$M_{D}$ & Ground station rotor mass & $2000 \mathrm{~kg}$ \\
$u_{T_{\max }}$ & Saturation on traction actuator & $65 \mathrm{kN}$ \\
$v_{w}$ & Wind speed & $10 \mathrm{~m} / \mathrm{s}$ \\
$\operatorname{Re}$ & Reynolds number & $1.7 \times 10^{6}$ \\
\hline
\end{tabular}

speed $v_{w}=10 \mathrm{~m} / \mathrm{s}$, the tether speed during the production phase $\dot{r}_{\text {prod }}$ and during the recovery one $\dot{r}_{\text {rec }}$ are found numerically offline. One gets $\dot{r}_{r e c}=-0.52 v_{w}$ and $\dot{r}_{\text {prod }}=0.33 v_{w}$ which is not the optimal value given by the main theory of simple kite [Loyd1980]. This is because the colinearity condition of $v_{k}$ and $T$ is not satisfied in vertical trajectories. By simulating this system at a wind speed $v_{w}=10 \mathrm{~m} / \mathrm{s}$, we get the net output power produced during a full cycle as a function of $X$ during the production phase (Fig. 1.16). $X$ is then set to 0 during the recovery phase. The maximum net output power equals $59.23 \mathrm{~kW}$ for $X=4.3$. The proposed control strategy will therefore use this nominal production cycle and vary the spin ratio $X$ between 0 and 4.3 in order to stabilize the desired power produced.

For this nominal production cycle, the energetic performance is $1.48 \mathrm{~kW} / \mathrm{m}^{2}$ which is consistent with $1.25 \mathrm{~kW} / \mathrm{m}^{2}$ found in [Milutinovic2015] where a similar sized system is used.

Note that we do not consider here the motor consumption that actuates the Magnus rotor. An estimation of this consumption can be computed as follows: Based on the $C_{M_{z}}$ Magnus parameter of [Perkovic2013] for $\mathrm{Re}=10^{6}$, the torque exerted on the Magnus rotor is:

$$
M_{z}=0.5 \rho \pi R^{2} L_{m} v_{a}^{2} C_{M_{z}}
$$

and the motor power consumption can be calculated by:

$$
P_{M}=\omega M_{z}=v_{w} \frac{X}{R} M_{z}=0.5 X \rho \frac{\pi}{2} S v_{w}^{3} C_{M_{z}}
$$

If one considers a spin ratio of $X=4.3$ and $v_{w}=10 \mathrm{~m} / \mathrm{s}$, one can estimate $C_{M_{z}}=$ 0.0055 , and $P_{M}=910 \mathrm{~W}$ for the production phase (61.2\% of the time). The consumption of the motor is $556.7 \mathrm{~W}$ for the whole cycle which is $0.9 \%$ of the 59.23 $\mathrm{kW}$ produced. 
Fig. 1.16 The variation of the net output power as a function of the spin ratio $X$ during the production phase for the medium scale system. During the recovery phase, $X$ is set to zero. Wind speed $v_{w}=10 \mathrm{~m} / \mathrm{s}$.

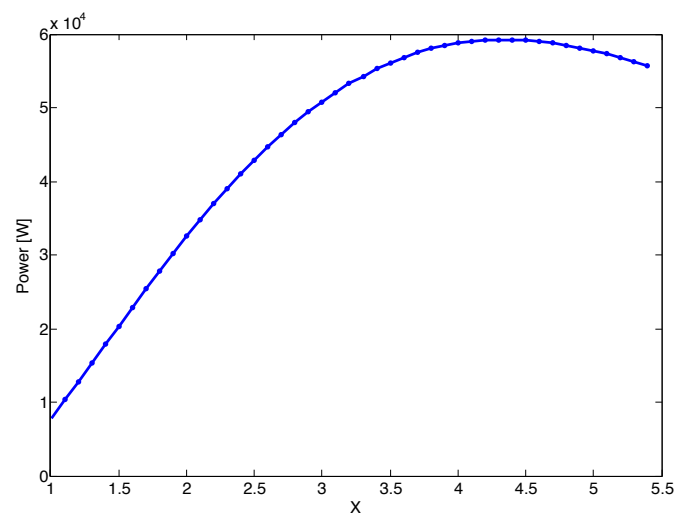

\subsubsection{Nominal Production Cycle}

In this section, the results of the nominal production cycle are presented. In order to have a smooth movement of the Magnus rotor, the reference tether length $r_{r e f}$ is filtered by $1 /\left(\tau_{R} s+1\right)^{2}$ with $\tau_{R}=2$ s.

The PID controller $K_{1}$ parameters are $K_{p}=8250 \mathrm{~N} / \mathrm{m}, K_{i}=1.32 \mathrm{~N} /(\mathrm{ms})$, $K_{d}=45 \times 10^{3} \mathrm{Ns} / \mathrm{m}$. We find that the apparent wind speed increases thanks to the temporal evolution of elevation angle $\beta$ (Fig. 1.17) which produces the cycle of Fig. 1.18 with a maximum of $v_{a}=14.26 \mathrm{~m} / \mathrm{s}$ in the production phase and $v_{a}=14.79 \mathrm{~m} / \mathrm{s}$ in the recovery phase. Following the simple kite theory, one can get an elevation angle $\beta=0$ for the recovery phase and $\beta=52.6 \mathrm{deg}$ for the production phase. This type of cycle is composed of the succession of transition phases between these two values of $\beta$. In Fig. 1.19, we show the temporal evolution of the tether length, tether tension and angular speed of the Magnus rotor. One can find the maximum tension on the tether is $T_{\max }=42.4 \mathrm{kN}$, the maximum angular speed $\omega_{\max }=49.02 \mathrm{rad} / \mathrm{s}$. The production phase reel-out speed is $3.3 \mathrm{~m} / \mathrm{s}$ with an overshoot measured at $8 \mathrm{~m} / \mathrm{s}$, the recovery phase speed is set to $-5.2 \mathrm{~m} / \mathrm{s}$, without any observed overshoot. Omnidea's current system cannot completely meet these values since the announced maximum force has been $5 \mathrm{kN}$ with a maximum angular speed of $9.42 \mathrm{rad} / \mathrm{s}$ [awec2015]. 

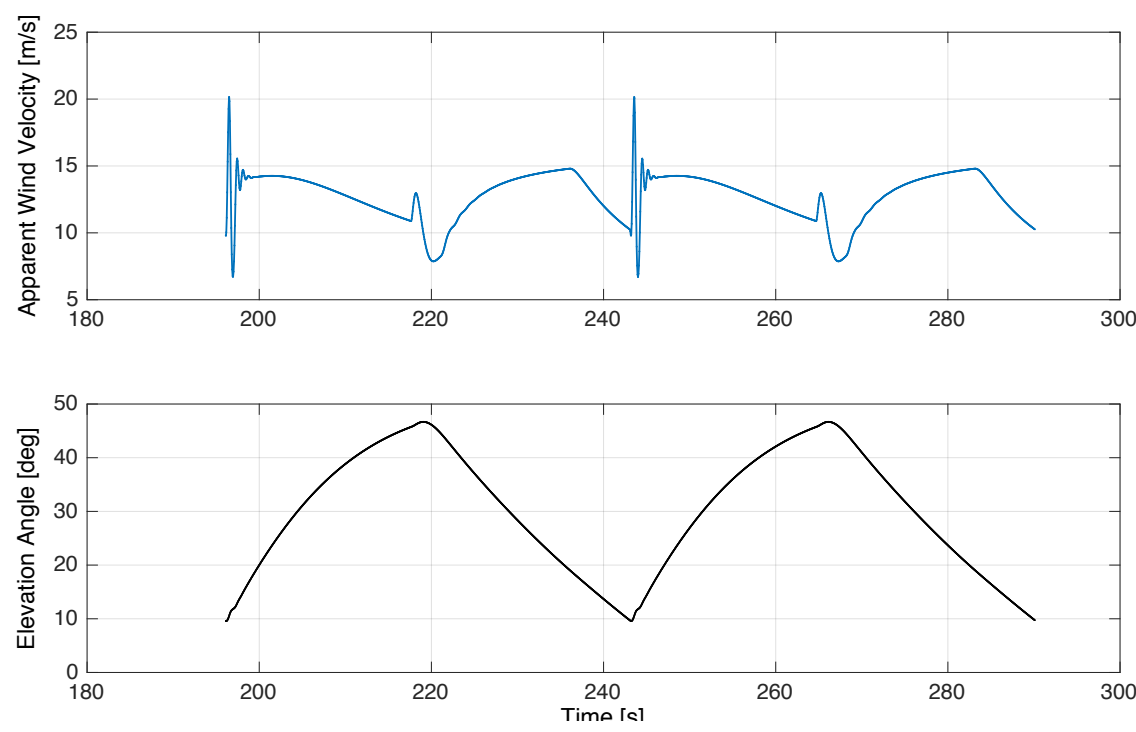

Fig. 1.17 Apparent wind speed in [m/s] (top) and elevation angle $\beta$ in [deg] (bottom) as function of time for the medium scale system. Wind speed $v_{w}=10 \mathrm{~m} / \mathrm{s}$.

Fig. 1.18 The production cycles of the medium scale system. Wind speed $v_{w}=10 \mathrm{~m} / \mathrm{s}$. The direction of the arrows indicates the movement of the Magnus rotor: Green for the production phase and red for the recovery phase.

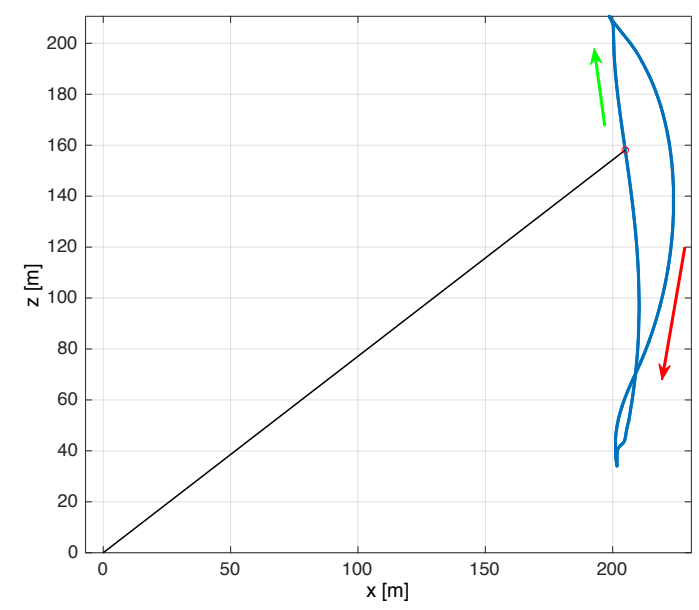

\subsubsection{Energy Control}

In this section, the complete control strategy has been applied. To find the control parameters of the controller $K_{2}$ (PD controller), we have chosen the increasing line slope of Fig. 1.16 between $X=1$ and $X=4.3$. The control parameters are then $K_{p}=6.4 \times 10^{-3} N^{-1}$ and $K_{d}=6.4 \times 10^{-3} \mathrm{~s} / \mathrm{N}$. One can clearly see the performance of the proposed control strategy (Fig. 1.20). The produced power will follow the 

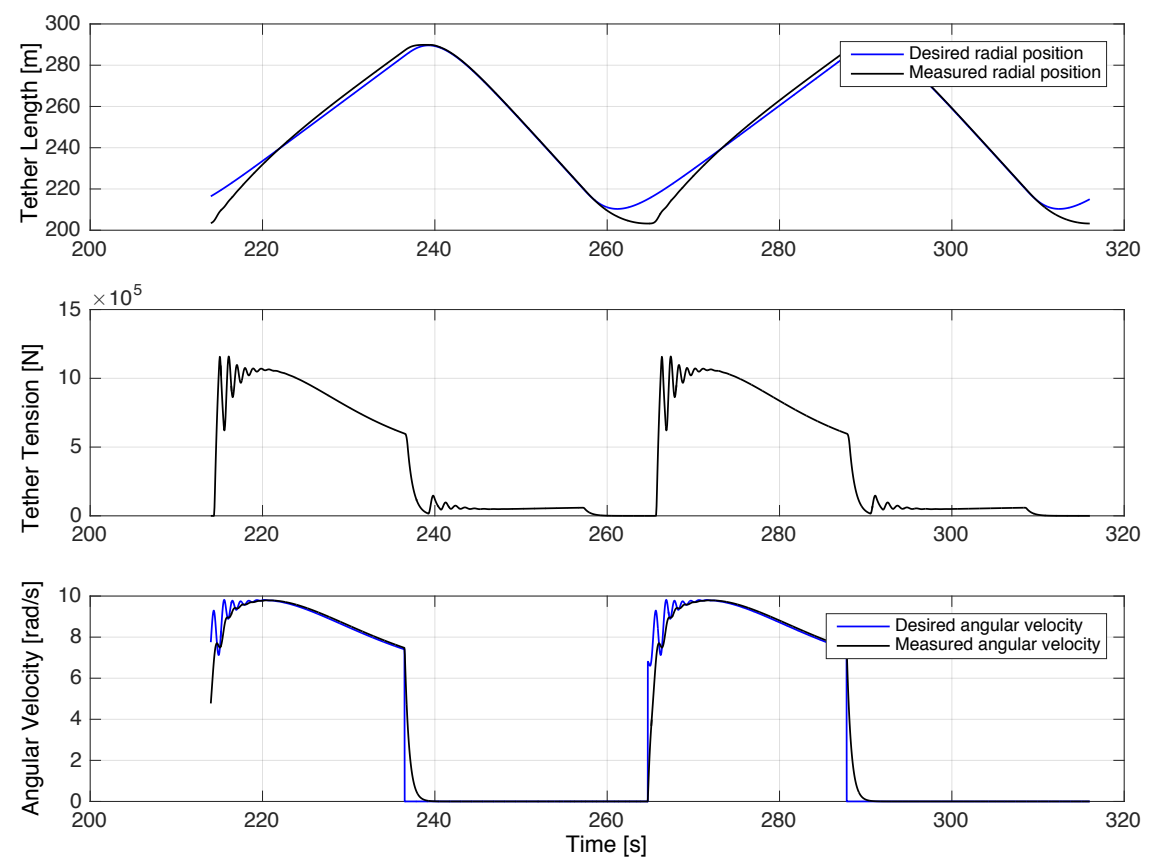

Fig. 1.19 Tether length, tether tension and the angular speed of the Magnus rotor as function of time for the medium scale system. Wind speed $v_{w}=10 \mathrm{~m} / \mathrm{s}$.

desired one even in the presence of noise on the wind speed. It is worth noting that if the output of PD is saturated, one can simply apply a very large reference to achieve the nominal production cycle, with $X=4.3$ throughout the production phase.

\subsubsection{Energy Control with Real Wind Data}

The energy control algorithm is also applied using real wind data taken on October 2015 at the Bard station of the Loire region in France [Bard2015] $]^{3}$. Only the wind magnitude is considered given that we are studying the movement in the vertical plane. The wind speed varies from $7 \mathrm{~m} / \mathrm{s}$ to $20 \mathrm{~m} / \mathrm{s}$. Three power reference levels are considered (Fig. 1.22):

- $P_{r e f}=20 \mathrm{~kW}$ : In this case, the system succeeds to track the desired power reference by limiting the energy produced even in the presence of wind turbulence. These variations in the wind speed generate a traction force that exceeds the on-

\footnotetext{
${ }^{3}$ The measurement sampling period is five seconds.
} 

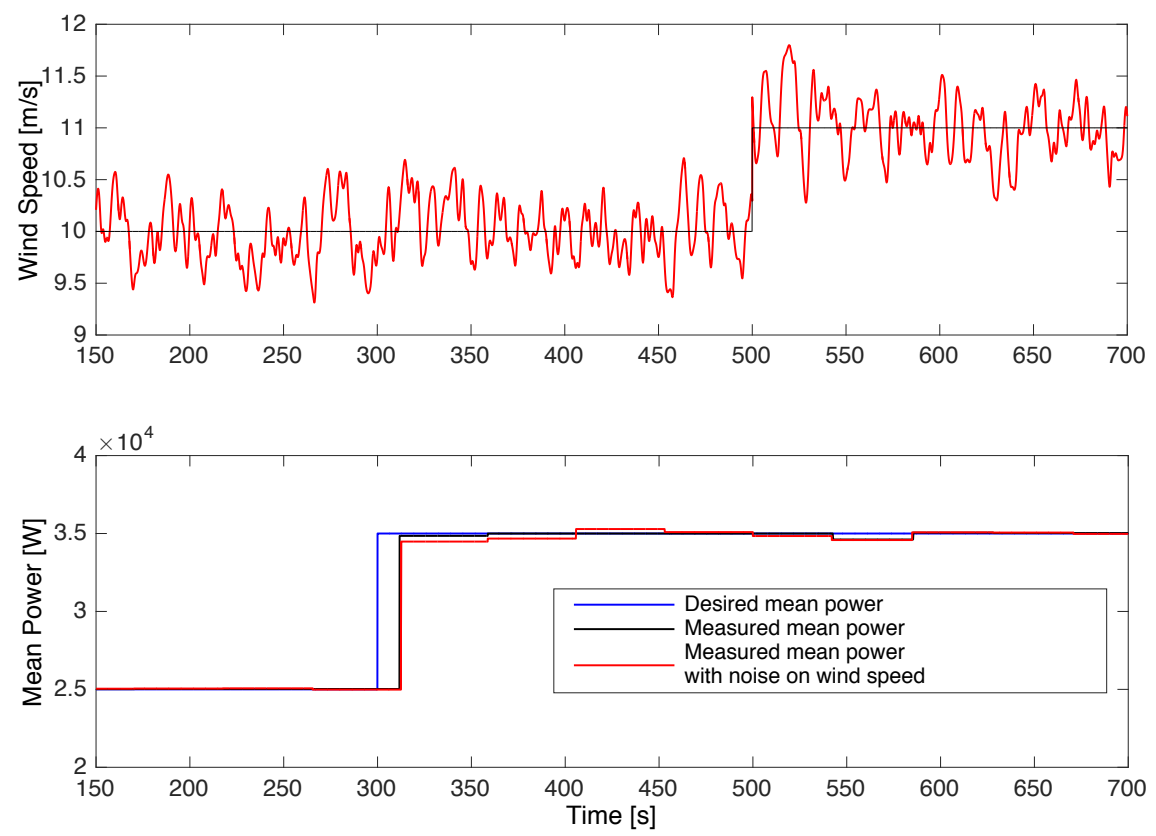

Fig. 1.20 A noise is added to the wind speed to test the performance of the control strategy (top). The net output power produced as a function of the desired level of power reference with a change in wind speed (from $10 \mathrm{~m} / \mathrm{s}$ to $11 \mathrm{~m} / \mathrm{s}$ ) for the medium scale system (bottom).

ground generator saturation which causes an error on the control of $r$ but does not affect the power produced.

- $P_{\text {ref }}=50 \mathrm{~kW}$ : The system succeeds to track the desired power reference when the available wind speed is enough. A short-term storage system can be used to ensure that the system catches up with the remaining energy of the previous cycle and thus obtains the desired average power in the presence of such fast changes in the wind.

- $P_{r e f}=90 \mathrm{~kW}$ : In this case, the wind speed is not high enough and the desired power reference is never attained. 

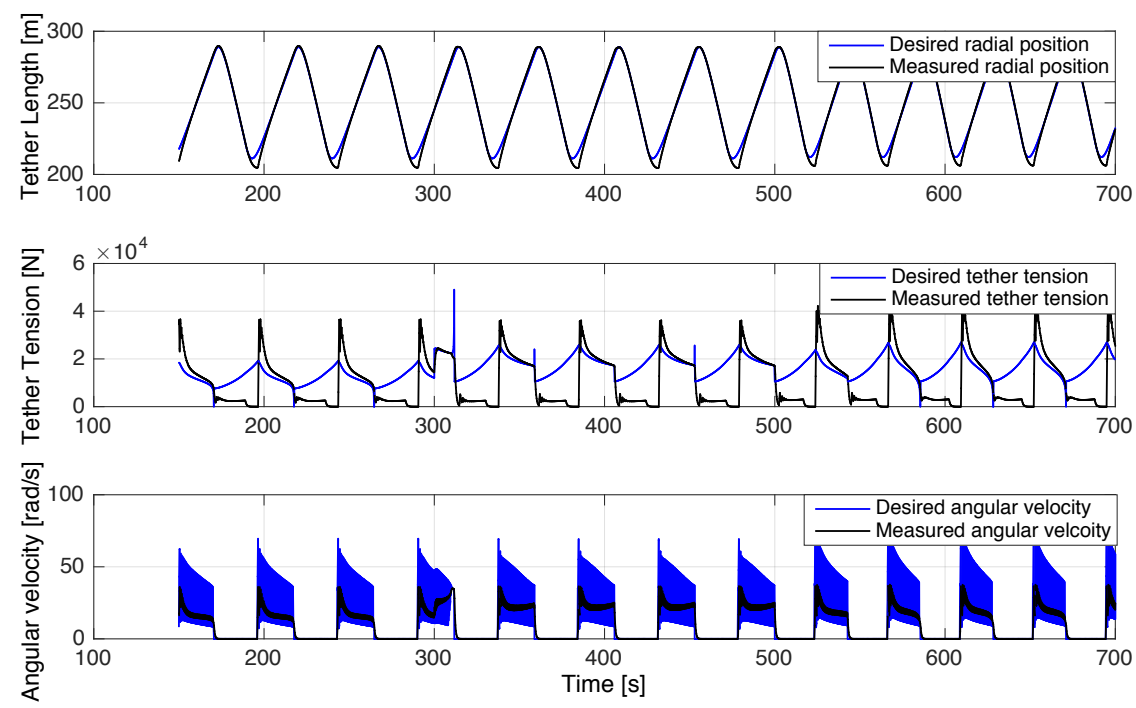

Fig. 1.21 Tether length, tether tension and the angular speed of the Magnus rotor as function of time in absence of noise for the medium scale system. The oscillations in the rotation speed are due to the choice of the control parameters. Wind speed $v_{w}=10 \mathrm{~m} / \mathrm{s}$.

\subsection{Numerical Application to a Future MW Scale System}

In order to evaluate the feasibility and scaling behavior of this kind of system, numerical simulations for a MW scale system have been performed. Its parameters are listed in Table 1.3 and correspond to a factor 25 from the medium scale system of the previous section. For $v_{w}=10 \mathrm{~m} / \mathrm{s}$, Reynolds number reaches $\operatorname{Re}=8.6 \times 10^{6}$.

Table 1.3 Parameters of the MW scale Magnus rotor

\begin{tabular}{lll}
\hline Symbol & Name & Value \\
\hline$M_{M}$ & Mass of airborne subsystem & $1.133 \times 10^{4} \mathrm{~kg}$ \\
$R$ & Magnus rotor radius & $6.25 \mathrm{~m}$ \\
$L_{m}$ & Magnus rotor length & $80 \mathrm{~m}$ \\
$\rho_{g}$ & Buoyant gas density & $0.95 \mathrm{~kg} / \mathrm{m}^{3}$ \\
$\rho_{\text {air }}$ & Air density & $1.225 \mathrm{~kg} / \mathrm{m}^{3}$ \\
$M_{l}$ & Mass per tether length & $5 \mathrm{~kg} / \mathrm{m}$ \\
$M_{D}$ & Magnus rotor mass & $50000 \mathrm{~kg}$ \\
$u_{T_{\max }}$ & Saturation on traction actuator & $2 \times 10^{6} \mathrm{~N}$ \\
$v_{w}$ & Wind speed & $10 \mathrm{~m} / \mathrm{s}$ \\
$\operatorname{Re}$ & Reynolds number & $8.6 \times 10^{6}$ \\
\hline
\end{tabular}



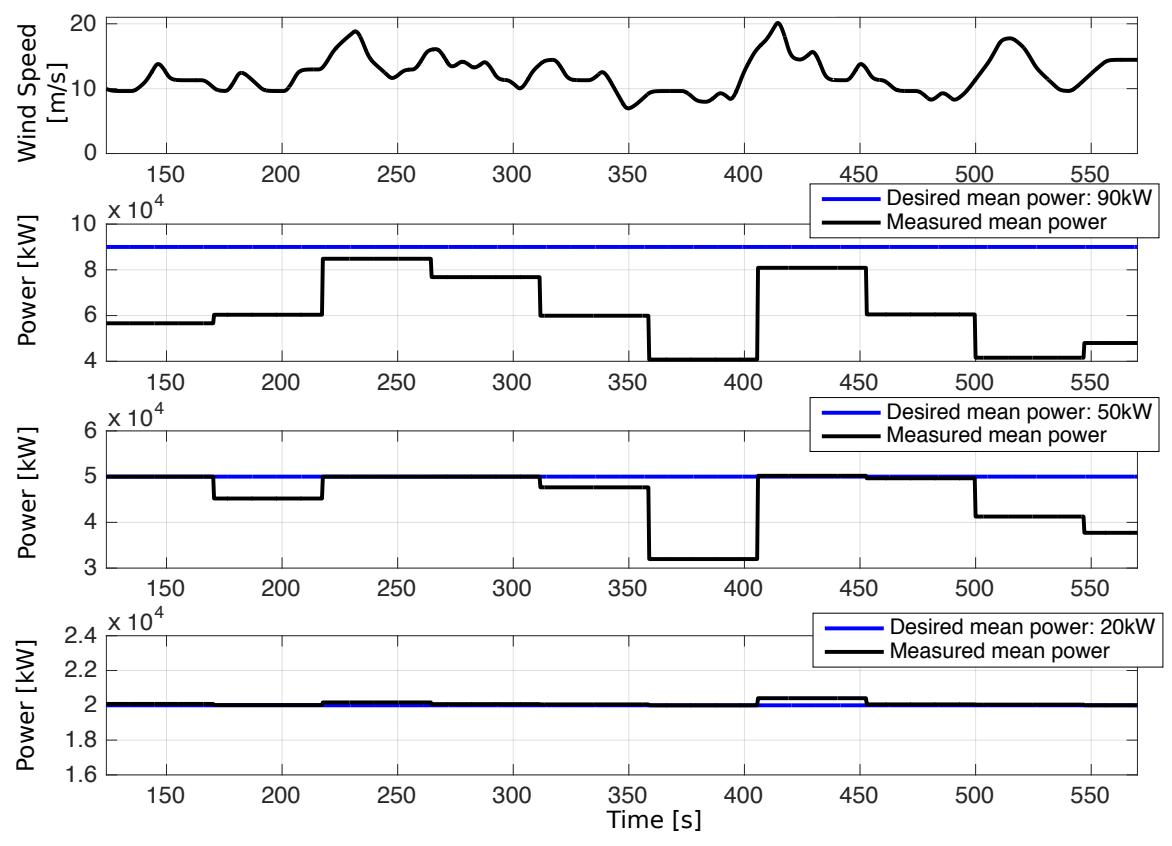

Fig. 1.22 Energy control of the medium scale system. Real wind speed data is used (top). Three levels of power reference are considered: $20 \mathrm{~kW}, 50 \mathrm{~kW}$, and $90 \mathrm{~kW}$. The wind speed varies from $7 \mathrm{~m} / \mathrm{s}$ to $20 \mathrm{~m} / \mathrm{s}$.

By scaling up, the volume of the Magnus rotor increases with the cube of the rotor dimension while the mass increases with the square, because it is related to the Magnus rotor surface. The gas used to fill the Magnus rotor can be more dense, keeping the whole system lighter-than-air without using pure Helium. As in the previous section, the cycle parameters are set in order to get a nominal production cycle with vertical trajectories. We have determined the feasibility regions for $r_{\min }=$ $200 \mathrm{~m}$ and $r_{\max }=300 \mathrm{~m}$. For a wind speed $v_{w}=10 \mathrm{~m} / \mathrm{s}$, the tether speed in the production phase $\dot{r}_{\text {prod }}$ and in the recovery phase $\dot{r}_{\text {rec }}$ are found numerically offline. One gets $\dot{r}_{\text {prod }}=0.31 v_{w}$ and $\dot{r}_{\text {rec }}=-0.46 v_{w}$ which are slightly different form those found for the medium scale system.

By simulating this system at a wind speed $v_{w}=10 \mathrm{~m} / \mathrm{s}$, with the same method of the previous section, the net output power is found to be $1.37 \mathrm{MW}$ for $X=4.3$, which corresponds to an energetic performance of $1.37 \mathrm{~kW} / \mathrm{m}^{2}$. This is consistent with the results of the medium scale system $1.48 \mathrm{~kW} / \mathrm{m}^{2}$ and $1.25 \mathrm{~kW} / \mathrm{m}^{2}$ found in [Milutinovic2015].

PID controller $K_{1}$ parameters are $K_{p}=5.16 \times 10^{5} \mathrm{~N} / \mathrm{m}, K_{i}=82.5 \mathrm{~N} / \mathrm{ms}, K_{d}=$ $2.81 \times 10^{6} \mathrm{Ns} / \mathrm{m}$. These control parameters are chosen empirically.

In Fig. 1.23, tether length, tether tension and the angular speed of the Magnus rotor as function of time are shown. One can find the maximum tension in the tether 

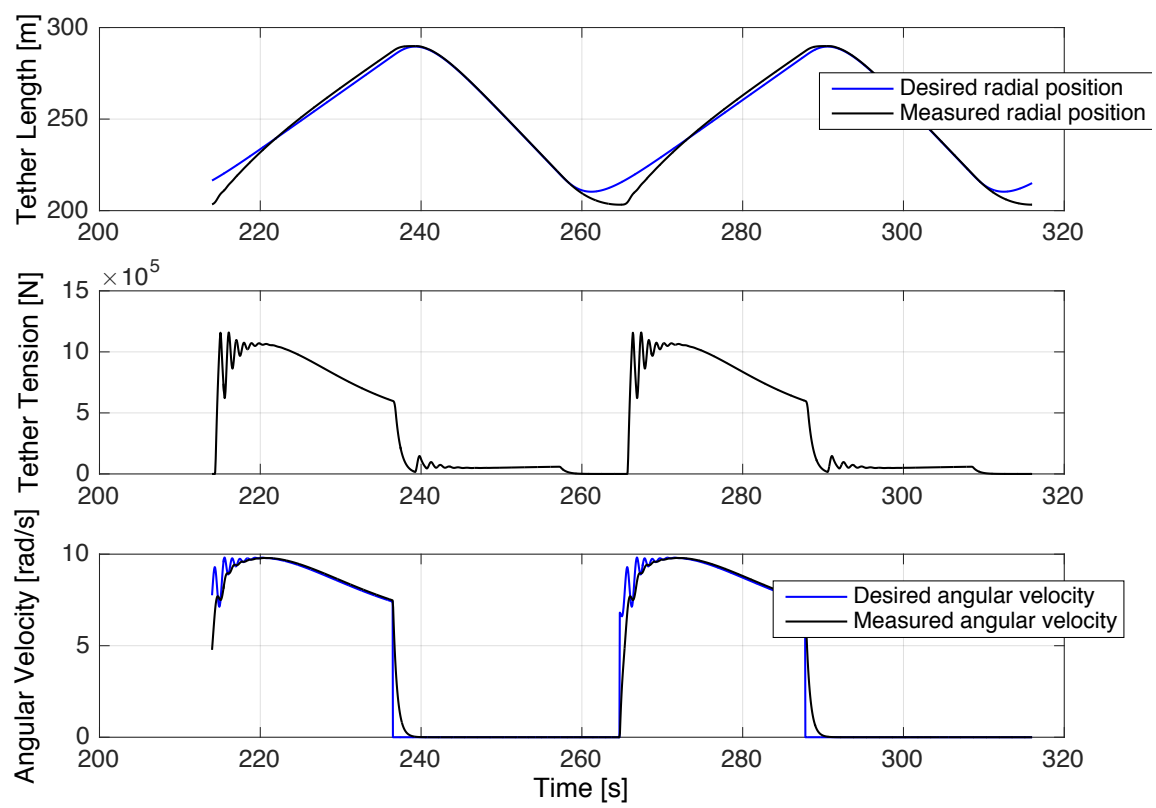

Fig. 1.23 Tether length, tether tension and the angular speed of the Magnus rotor as function of time for the MW scale system. Wind speed $v_{w}=10 \mathrm{~m} / \mathrm{s}$.

$T_{\max }=1.16 \times 10^{6} \mathrm{~N}$ and the maximum angular speed $\omega_{\max }=9.8 \mathrm{rad} / \mathrm{s}$. The production phase reel-out speed is $3.1 \mathrm{~m} / \mathrm{s}$ with an overshoot measured at $7.4 \mathrm{~m} / \mathrm{s}$, the recovery phase reel-in speed is set to $-4.6 \mathrm{~m} / \mathrm{s}$, without any observed overshoot.

In Fig. 1.24, one can see the vertical trajectory of the MW scale system. We also present a comparison with an equivalent conventional wind turbine. Even though the Magnus effect-based system is less efficient to capture mechanical energy from wind, it produces the same amount of power as an $80 \mathrm{~m}$ diameter wind turbine (around $1.4 \mathrm{MW}$ for $10 \mathrm{~m} / \mathrm{s}$ wind speed) since it works on a larger area. In other words, an $80 \mathrm{~m}$ diameter wind turbine works on $5000 \mathrm{~m}^{2}$ with a power coefficient $c_{p}=0.45$ where the Magnus effect-based system works on $13940 \mathrm{~m}^{2}$ with a power coefficient $c_{p}=0.157$. With the same method used in Sect. 1.5, the Magnus motor consumption can be estimated by $P_{M}=22.7 \mathrm{~kW}$ for $C_{M}=0.0055, X=4.3$ and $v_{w}=10 \mathrm{~m} / \mathrm{s}$. Knowing that production phase is $59 \%$ of the time, the net output power of the Magnus motor over the whole cycle is $13.56 \mathrm{~kW}$ which is about $1 \%$ of the power produced. 

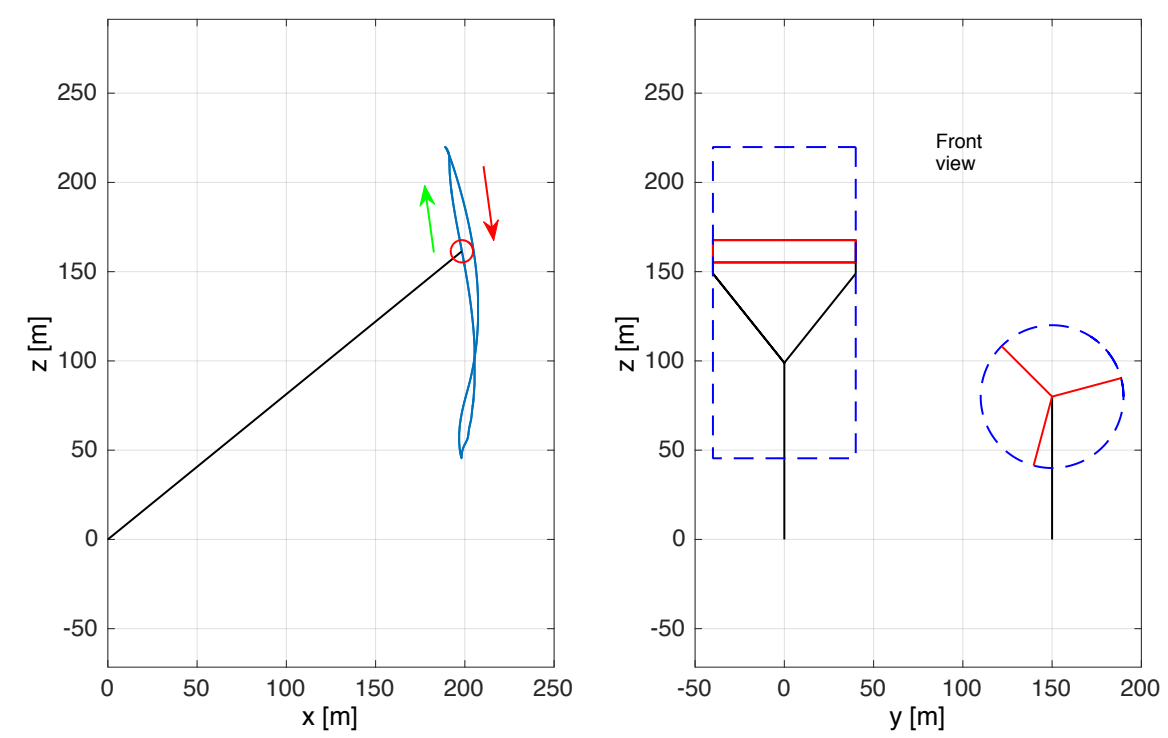

Fig. 1.24 The production cycles of the MW scale system. The direction of the arrows indicates the movement of the Magnus rotor: Green for the production phase and red for the recovery phase. On the right side figure, the MW scale system and an equivalent conventional wind turbine are compared.

\subsection{Conclusions and Perspectives}

The control of an airborne wind energy system based on a Magnus rotor have been presented. The small scale system wind tunnel experiments have enabled us to test different aspects of the system and to validate part of the proposed control strategy. The Magnus effect-based model was validated for a spin ratio ranging from 1 to approximately 2.3. Our goal for a future work is the experimentation of such models for a spin ratio greater than 5.5 in order to increase efficiency. The small size of our wind tunnel does not allow to reach tether reel-out speeds that would achieve the simulated performance of $1.48 \mathrm{~kW} / \mathrm{m}^{2}$, but faster dynamics of the actuators would allow to achieve vertical trajectories.

Nominal production cycles have been studied for a medium scale systems with vertical trajectories achieving the simulated performance of $1.48 \mathrm{~kW} / \mathrm{m}^{2}$. For these vertical trajectories, the simple kite case described in [Loyd80] cannot be considered since the translation velocity of the Magnus rotor $\mathbf{v}_{\mathbf{k}}$ is not colinear with the traction force $\mathbf{T}$. This type of $2 \mathrm{D}$ cycles has to be formally studied in order to optimize its performance. The complete control strategy has been applied. The system succeeds to track the desired power reference even in the presence of wind turbulence. This strategy can be applied in a future work on other types of AWE systems by adapting the control variables. 
Finally, in order to study the feasibility of a MW scale system, numerical simulation has been performed. The production cycle gives $1.38 \mathrm{~kW} / \mathrm{m}^{2}$ based on vertical trajectories. The problem of scalability due to the structure's resistance to important forces is not treated here and must be addressed later to ensure that the Magnus rotor can withstand such mechanical stress.

Acknowledgements The authors of this chapter would like to thank the technical staff of Gipsalab and the trainees Azzam Alwann, Alexandre Kajiyama and Pierre Estadieu. They also thank the editor and the anonymous reviewers for their constructive comments, which helped to improve the quality of the chapter. 